\title{
A Patient with a Pancreatic Endocrine Tumor develops Chronic Schizophrenia: Report of a Case
}

\section{Sean Hood}

FRANZCP, School of Psychiatry and Neuroscience, University of Western Australia, Perth, Australia

\section{Introduction}

The pancreatic endocrine tumors are heterogeneous group of pancreatic neoplastic growths that can be biologically active in nearly one third of cases; these tumors represent $1-2 \%$ of pancreatic neoplasia [1]. Traditionally, these tumors have been named after their active biological secretions, such as insulinoma, gastrinoma and others. In 2011 Mussig et al. [2] reported pancreatic endocrine tumors as rare entities with an annual incidence rate of $<100,000$ [2]. The majority of pancreatic endocrine tumors are sporadic but they can be hereditary if they are part of the syndrome of multiple endocrine neoplasia type 1, which is an autosomal dominant disorder [3]. It has been suggested that various endocrine disorders may intensify the possibility of developing psychiatric illnesses. For example, in the majority of reported cases the psychiatric manifestations of thyroid endocrine dysfunction are well established [4]. The association of pancreatic endocrine tumors (PET) and chronic schizophrenia, however, is a rare event [5].

Schizophrenia is a severe and chronic psychiatric illness that is characterized by a significant long term decline in patient's functional performance due to psychotic phenomena including delusions, hallucinations and the associated negative syndrome. The cause of schizophrenia remains to be elucidated but the link between schizophrenia and pancreatic endocrine tumors remains hypothetical [5]. In this article we report a patient with a confirmed history of pancreatic endocrine tumor who developed chronic schizophrenia and who has been followed-up over a 13 year period.

\section{Case Report}

A 22-year-old single man who worked as a grocery packer was referred to a community psychiatric clinic in Perth, Australia by his primary care physician for cognitive and behavioral treatment of panic disorder (PD). His first panic attack occurred at work six months previously and he recalled feeling that blood was pounding in his head. He reported paraesthesia in his hands, tongue and lips followed by a pre-syncope sensation, headache, palpitations, and a fear of impending doom. The attack took seconds to reach a climax and resolved within a couple of minutes. Similar attacks had occurred about every fortnight since, usually in the mornings at work but occasionally at home. He described a loss of appetite, mild weight loss and decreased libido in recent months. There was no agoraphobic avoidance or past psychiatric history. There was no history of psychiatric illness in his family. His mother suffers from diabetes type 2 and hypothyroidism. He admitted that he had taken intravenous anabolic steroids with the sulfonylurea glipizide as part of a body building regimen for about $1-1 / 2$ years but stated firmly that he stopped the steroids injections about six months before the first panic attack.

Random blood glucose was low at $3.0 \mathrm{mmol} / \mathrm{L}$, and a glucose tolerance test performed the next day showed a fasting glucose of 2.2 $\mathrm{mmol} / \mathrm{L}$ which was $5.5 \mathrm{mmol} / \mathrm{L}$ and still rising after 2 hours. After referral to a tertiary hospital endocrine unit a prolonged fast precipitated a panic attack where the serum glucose level was low while the serum insulin level was high, raising a possibility that the patient suffered from a functioning pancreatic endocrine tumor. A CT scan of the abdomen was ordered and showed a well-encapsulated mass in the body of the pancreas. The patient had an Endoscopic Ultrasound examination of the pancreas (EUS) that showed a well circumscribed mass in the body of the pancreas, the mass was $42 \mathrm{~mm}$ in diameter, it had a homogeneous echo texture and no evidence of local invasion was detected. The initial provisional clinical diagnosis was a functioning pancreatic endocrine tumor that correlated with the patient's hypoglycemic pre-syncope episodes and prolonged periods of fasting hypoglycemia. The patient underwent a laparotomy enucleation of the pancreatic tumor; the pathology confirmed the presence of pancreatic tumor cells arranged in gyriform and glandular pattern. The tumor was composed of solid and cystic areas, the cystic spaces lined with cuboidal and columnar epithelium. The tumor was vascular. The immunohistochemistry showed the tumor cells to stain positively with synaptophysin, which is a neuroendocrine marker. The final confirmed diagnosis confirms is a pancreatic endocrine tumor (Figures 1-3).

After the operation, the patient needed to be admitted to the surgical unit again to manage a pancreatic fistula and a pancreatic fluid collection; he underwent endoscopic drainage of the fluid collection and was discharged to the care of his local general practitioner. The patient was later referred to the community psychiatric clinic one year after the discharge from the surgical ward when he first experienced psychotic symptoms including paranoid delusions, persecutory and referential ideas from TV and radio, thought insertion, withdrawal and broadcasting consistent with a diagnosis of chronic schizophrenia. The patient needed several admissions to psychiatry hospital wards in Perth over 4 years to manage the psychotic symptoms and treat several

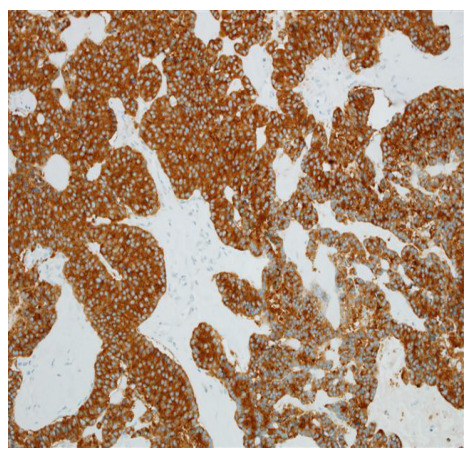

Figure 1: Neuroendocrine marker synaptophysin, demonstrating strong positive staining in tumor cell cytoplasm. (Synaptophysin IHC $\times 200$ ).

*Corresponding author: Sean Hood, MBBS, Sir Charles Gairdner Hospital, Perth, Australia, Tel: (+61 8) 9346 2140; E-mail: ahudaib@gmail.com

Received November 14, 2013; Accepted December 28, 2013; Published December 30, 2013

Citation: Hood S (2013) A Patient with a Pancreatic Endocrine Tumor develops Chronic Schizophrenia: Report of a Case. J Clin Case Rep 4: 328. doi:10.4172/2165 7920.1000328

Copyright: (c) 2013 Hood S. This is an open-access article distributed under the terms of the Creative Commons Attribution License, which permits unrestricted use, distribution, and reproduction in any medium, provided the original author and source are credited. 


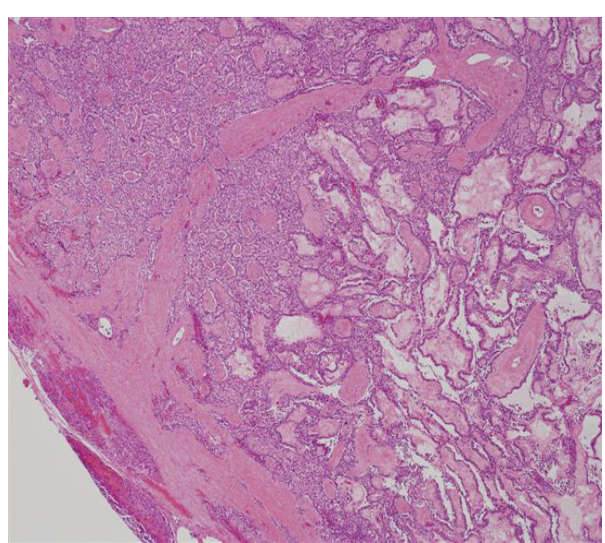

Figure 2: Tumor demonstrating trabecular/gyriform architecture, with areas of hyalinized oedematous and myxoid connective tissue cores. No amyloid was identified with Congo red staining. $(H \& E \times 100)$.

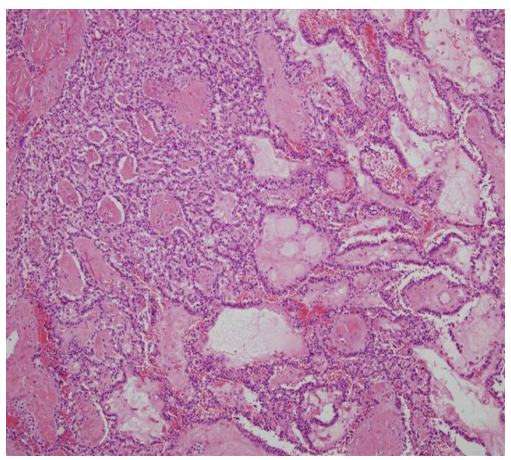

Figure 3: Encapsulated pancreatic endocrine neoplasm, with a small amount of compressed residual pancreatic parenchyma at lower left. $(H \& E \times 40)$.

relapses of schizophrenia. The patient was linked with a psychiatric community team to encourage compliance with the long term depot antipsychotic treatment. Recently reviewed by us again over a decade postoperatively, the patient is doing well with his regular psychiatry community follow up with no recent relapses and no reported episodes of presyncope.

\section{Discussion}

Incidental co-occurrence of psychiatric disorders in patients with endocrine dysfunctions is possible. Nevertheless, the emergence of chronic schizophrenia in a patient with confirmed diagnosis of pancreatic endocrine tumor is rare. In 2005, Kito et al. [5] reported a case where an association between chronic schizophrenia and a hereditary nonfunctioning islet cell pancreatic tumor (part of Multiple Endocrine Neoplasia (MEN1) was noted [5]. In the current case, however, the chronic schizophrenia is associated with a sporadic pancreatic endocrine tumor.

The pancreatic endocrine tumors were previously called "islet cell tumors of pancreas". This nomenclature is no longer used in recent publications due to accumulated evidence that they originate from ductal pancreatic stem cells [6]. It is believed that endocrine cells of the pancreas differentiate from the primitive epithelial ductal cells that produce the islets of Langerhans and account for the multidifferentiation capability of these tumors, reflecting its various spectra of hormone secretions.

A substantial percentage of functioning pancreatic endocrine tumors represent insulinomas that tend to present clinically earlier and at a smaller size due to the hormonal status wherein insulin is over secreted. This causes symptoms of fasting hypoglycemia including syncopal episodes and panic attacks [1]. The hormonal profile of other functioning pancreatic endocrine tumors is recognized and is linked to well-known clinical syndromes reported in current literature. The syndromic features of schizophrenia as a psychiatric illness, in contrast, have rarely been reported as an association.

The causation of schizophrenia as a chronic debilitating mental illness remains unclear. Two hypotheses are well described, namely the dopamine and serotonin models, the dopamine model forms the basis for the current antipsychotic regimens and has proved to be valuable in psychiatry clinical practice [7]. The hormonal basis of schizophrenia has been an interesting field of study for many years and hence we report this case to highlight the possibility that the super-physiological action of hormones secreted by pancreatic tumors may shed new light on the etiology of schizophrenia and related syndromes.

Our case is noticeable not only because it reports a rare association between a pancreatic endocrine tumor and chronic schizophrenia but also because it also raises new questions on the hormonal basis of this chronic mental illness.

\section{References}

1. Hruban RH, Pitman MB, Klimstra DS (2007) Tumors of the pancreas. AFIP Atlas of tumor pathology. (4th edition), Washington, American Registry of Pathology.

2. Müssig K, Dudziak K, Horger M, Anlauf M, Goretzki PE (2011) [Diagnostics and treatment in functional pancreatic neuroendocrine tumours]. Dtsch Med Wochenschr 136: 1319-1330.

3. Shibata C, Funayama Y, Sasaki I (2008) Pancreatic endocrine tumors: epidemiology, pathology, pathophysiology, and diagnosis. Diseases of the pancreas: current surgical therapy. Berlin, Springer.

4. Smith CK, Barish J, Correa J, Williams RH (1972) Psychiatric disturbance in endocrinologic disease. Psychosom Med 34: 69-86.

5. Kito S, Nakajima T, Yamadera H, Koga Y, Kosugi S, et al. (2005) Multiple endocrine neoplasia type 1 presenting as psychosis. Am J Psychiatry 162 : 810-811.

6. Klöppel G, Anlauf M (2006) Pancreatic Endocrine Tumors. Pathology Case Reviews.

7. Shin JK, Malone DT, Crosby IT, Capuano B (2011) Schizophrenia: a systematic review of the disease state, current therapeutics and their molecular mechanisms of action. Curr Med Chem 18: 1380-1404. 